\title{
Musclin Is Related to Insulin Resistance and Body Composition, but Not to Body Mass Index or Cardiorespiratory Capacity in Adults
}

\author{
Yeliana L. Sánchez ${ }^{1}$, Manuela Yepes-Calderón ${ }^{1}$, Luis Valbuena ${ }^{1,2}$, Andrés F. Milán ${ }^{1}$, María C. Trillos-Almanza ${ }^{1}$, \\ Sergio Granados ${ }^{1}$, Miguel Peña ${ }^{1}$, Mauricio Estrada-Castrillón ${ }^{3}$, Juan C. Aristizábal ${ }^{1}$, Raúl Narvez-Sanchez ${ }^{1}$, \\ Jaime Gallo-Villegas ${ }^{1,4}$, Juan C. Calderón ${ }^{1}$
}

${ }^{1}$ Physiology and Biochemistry Research Group-PHYSIS, Faculty of Medicine, University of Antioquia; ${ }^{2}$ Indeportes Antioquia; ${ }^{3}$ Pablo Tobón Uribe Hospital; ${ }^{4}$ Sports Medicine Postgraduate Program, and GRINMADE Research Group, SICOR Center, Faculty of Medicine, University of Antioquia, Medellin, Colombia

Background: We studied whether musclin function in humans is related to glycemic control, body composition, and cardiorespiratory capacity.

Methods: A cross-sectional study was performed in sedentary adults with or without metabolic syndrome (MS). Serum musclin was measured by enzyme-linked immunosorbent assay. Insulin resistance (IR) was evaluated by the homeostatic model assessment (HOMA-IR). Body composition was determined by dual-energy X-ray absorptiometry and muscle composition by measuring carnosine in the thigh, a surrogate of fiber types, through proton magnetic resonance spectroscopy. Cardiorespiratory capacity was assessed through direct ergospirometry.

Results: The control $(n=29)$ and MS $(n=61)$ groups were comparable in age (51.5 \pm 6.5 years old vs. $50.7 \pm 6.1$ years old), sex ( $72.4 \%$ vs. $70.5 \%$ women), total lean mass $(58.5 \% \pm 7.4 \%$ vs. $57.3 \% \pm 6.8 \%)$, and peak oxygen consumption $\left(\mathrm{VO}_{2 \text { peak }}\right)(31.0 \pm 5.8$ $\mathrm{mL} \mathrm{O}_{2} . / \mathrm{kg}$.min vs. $\left.29.2 \pm 6.3 \mathrm{~mL} \mathrm{O} / \mathrm{kg} . \mathrm{min}\right)$. Individuals with MS had higher body mass index (BMI) $\left(30.6 \pm 4.0 \mathrm{~kg} / \mathrm{m}^{2}\right.$ vs. $27.4 \pm$ $3.6 \mathrm{~kg} / \mathrm{m}^{2}$ ), HOMA-IR (3.5 [95\% confidence interval, CI, 2.9 to 4.6 ] vs. 1.7 [95\% CI, 1.1 to 2.0$]$ ), and musclin (206.7 pg/mL [95\% CI, 122.7 to 387.8$]$ vs. $111.1 \mathrm{pg} / \mathrm{mL}$ [95\% CI, 63.2 to 218.5$])$ values than controls $(P<0.05)$. Musclin showed a significant relationship with HOMA-IR ( $\beta=0.23 ; 95 \% \mathrm{CI}, 0.12$ to $0.33 ; P<0.01$ ), but not with $\mathrm{VO}_{2 \text { peak }}$, in multiple linear regression models adjusted for age, sex, fat mass, lean mass, and physical activity. Musclin was significantly associated with insulin, glycemia, visceral fat, and regional muscle mass, but not with $\mathrm{BMI}, \mathrm{VCO}_{2 \text { peak }}$, maximum heart rate, maximum time of work, or carnosine.

Conclusion: In humans, musclin positively correlates with insulinemia, IR, and a body composition profile with high visceral adiposity and lean mass, but low body fat percentage. Musclin is not related to BMI or cardiorespiratory capacity.

Keywords: Hormones; Musclin protein, human (OSTN); Metabolic syndrome; Insulin resistance; Muscle, skeletal; Body composition

Received: 14 May 2021, Revised: 27 July 2021, Accepted: 24 August 2021

Corresponding author: Juan C. Calderón

Physiology and Biochemistry Research Group-PHYSIS, Department of

Physiology and Biochemistry, Faculty of Medicine, University of Antioquia,

UdeA, Calle 70 No 52-21, Medellin, Colombia

Tel: $+57-604-2196035$, Fax: $+57-604-2638282$,

E-mail: jcalderonv00@yahoo.com

\section{Copyright $\odot 2021$ Korean Endocrine Society}

This is an Open Access article distributed under the terms of the Creative Commons Attribution Non-Commercial License (https://creativecommons.org/ licenses/by-nc/4.0/) which permits unrestricted non-commercial use, distribution, and reproduction in any medium, provided the original work is properly cited. 


\section{INTRODUCTION}

Skeletal muscle is a major regulator of metabolic glycemic control in healthy humans and in individuals with metabolic diseases, such as metabolic syndrome (MS): (1) both muscle contraction and insulin stimulate the transport of glucose into skeletal muscles [1-3]; (2) regional and global skeletal muscle mass and strength are inversely associated with the development of insulin resistance (IR) and MS [4-8]; and (3) muscles secrete myokines that are involved in regulating glucose transporter 4 (GLUT4) traslocation, glucose uptake, and fat oxidation in muscle, as well as hepatic glucose production [9-11].

Although many myokines may play a role in glycemic control in humans, and in the development of metabolic alterations associated with MS [12-14], musclin has recently attracted significant interest. It reduces glucose uptake and glycogen synthesis in myotubes and murine soleus muscles [11,15] by impairing the expression and phosphorylation of proten kinase B [10] and the regulation of GLUT4 [15]. Musclin is mainly produced by fast fiber types in murine models [16], the fiber type predominant in humans with conditions classically related to IR [17]. Noticeably, musclin is upregulated by palmitate and a high-fat diet $[15,18,19]$ and is downregulated by aerobic training in murine models $[19,20]$, an intervention known to improve glycemic metabolism in humans. The evidence, then, suggests that musclin may be involved in glycemic control and its alteration may have a role in the pathophysiology of IR in humans with MS. Information about the function of musclin in humans, nevertheless, is severely lacking.

More recently, it has also been proposed that this protein serves as an enhancer of physical capacity and a trophic factor for skeletal muscle $[21,22]$, and that it is upregulated by highintensity interval training [23] in mice, which itself is expected to protect against metabolic alterations and IR. This apparent controversy further encourages the need for further research to make progress on elucidating the role of musclin in humans.

In this study, we aimed to evaluate whether musclin is related to glycemic control, IR, and an altered body composition or to cardiorespiratory and physical capacity in humans with and without MS. Our aim is to offer new insights into the function of musclin in humans while addressing the apparent controversy raised by some murine studies. We hypothesize that musclin is related to variables indicating metabolic alterations and is increased in subjects with MS, but is not directly associated with parameters of cardiorespiratory endurance.

\section{METHODS}

\section{Study design}

This cross-sectional study recruited individuals of both sexes who were 40 to 60 years old. Volunteers were excluded if they were on vitamin D supplementation, received insulin or insulinsecretion-promoting drugs, had diseases that prevented them from performing an ergospirometry test, history of cardiovascular or cerebrovascular events, chronic respiratory diseases, systemic inflammatory conditions, diabetes mellitus, cancer, or were vegetarian or pregnant.

Participants were recruited between March 2017 and February 2019, in Medellin, Colombia. All of them underwent a complete medical history and physical examination, as well as biochemical tests. Based on that, the subjects were classified as having MS or not (controls), according to the internationally accepted criteria [24]. The waist circumference cutoff points were set according to the recommendation for the Colombian population [25]. All subjects with MS had IR, as defined by a homeostatic model assessment of insulin resistance (HOMA-IR) greater than 2.25 [25]. Most patients with MS also participated in the intraining-MET clinical trial [26].

After being assigned into study groups, the volunteers underwent cardiorespiratory fitness, body composition, and muscle composition studies, evenly scheduled at 3-day intervals over a period of 2 weeks (Fig. 1). All researchers performing the tests were blinded to the groups.

\section{Ethics approval}

All interventions were approved by the Research Ethics Committee of the Faculty of Medicine at the University of Antioquia (minutes 013 of September 26, 2013) and the Research Ethics Committee at IPS-Universitaria (minutes 097 of June 22, 2016) in Medellin (Colombia), in accordance with the Ethical Principles for Medical Research Involving Human Subjects outlined in the Declaration of Helsinki in 1975 and Resolution no. 8430 by the National Ministry of Health of Colombia issued in 1993. All participants gave written informed consent.

\section{Experimental procedures Complete medical history and physical examination}

Information regarding personal and familial clinical history, including nutritional aspects, habits, and socioeconomical conditions was recorded. Subjects were not enrolled in formal or structured physical activity programs in their leisure time, as verified using the Global Physical Activity Questionnaire (GPAQ) 


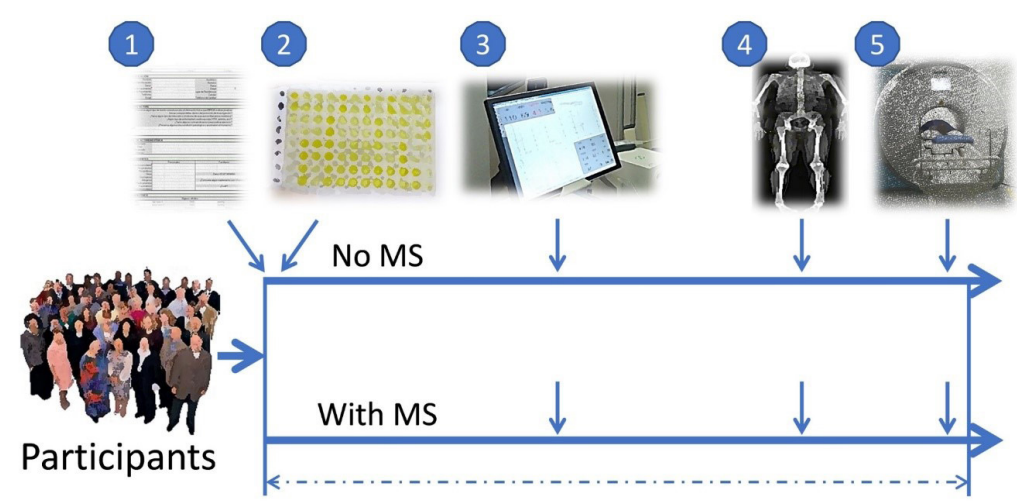

Two weeks

Fig. 1. Schematic representation of the study protocol. Participants were interviewed by two physicians and a complete medical history was recorded (1). If the participants did not meet any exclusion criteria, they were then scheduled for biochemical tests, including serum samples for musclin, all under fasting conditions (2). After being classified as with or without metabolic syndrome (MS) according to clinical and biochemical criteria, the participants underwent direct ergospirometry to evaluate their cardiorespiratory capacity (3). During the second week, after complete recovery from the physical test, they underwent dual-energy X-ray absorptiometry (4) and proton magnetic resonance spectroscopy (5) tests, to assess their body and muscle composition, respectively.

[27]. Blood pressure was measured in sitting and standing positions with a Welch Allyn DS44-11 manual sphygmomanometer (Welch Allyn, Skaneateles Falls, NY, USA) according to international recommendations [28]. Height was measured with a Seca 2013 stadiometer (Seca, Hamburg, Germany), and body weight was measured with an Omron HBF-510LA scale (Omron Healthcare Inc., Lake Forest, IL, USA) with an accuracy of $0.1 \mathrm{~kg}$. Body mass index (BMI) was calculated as weight $(\mathrm{kg}) / \mathrm{height}$ $(\mathrm{m})^{2}$. Waist circumference was measured with a fiberglass anthropometric tape at the intermediate point between the lower edge of the last rib and the iliac crest, in the horizontal plane.

\section{Biochemical tests}

Fasting venous blood samples were collected using serum separator tubes (BD Vacutainer 367815, BD, Franklin Lakes, NJ, USA). The samples were centrifuged at $1,300 \times g$ for 15 minutes at room temperature (Rotofix 32, Hettich, Westphalia, Germany). Fresh serum was used to measure circulating triglycerides, high-density lipoprotein, and glucose by spectrophotometry. Insulinemia was measured by direct chemiluminescence with an ADVIA Centaur CP Immunoassay System (Siemens, Munich, Germany). HOMA-IR was used to estimate IR since this minimally invasive technique was validated against the euglycemic clamp [29] and is widely used in clinical studies [30-32].

Serum aliquots were kept at $-80^{\circ} \mathrm{C}$ until processing. Fasting serum musclin was measured using a human musclin enzymelinked immunosorbent assay (ELISA) kit with a detection range between 15.6 and 1,000 pg/mL (catalog number LS-F7799,
LifeSpan BioSciences Inc., Seattle, WA, USA). The sensitivity is $6.7 \mathrm{pg} / \mathrm{mL}$ and it showed complete specificity for human recombinant musclin when tested against rat musclin, and human bone sialoprotein and bone morphogenetic protein 1. Spikeand-recovery assays with serum samples showed a recovery range between $93 \%$ and $101 \%$, according to the provider. Samples were brought to room temperature just before starting the assays, without using extra heat, and $100 \mu \mathrm{L}$ was loaded in duplicate into the wells of the microplate. The kits were always balanced with samples of subjects with and without MS and were processed by an investigator blinded to the group coding, according to the provider's instructions. The optical density of each well was determined on a plate reader (Varioskan Lux, Thermo Scientific, Waltham, MA, USA) at $450 \mathrm{~nm}$. The final values were reported in $\mathrm{pg} / \mathrm{mL}$ of musclin. Our standard curves always showed a fit with an $R^{2}$ over 0.98 , and our measurements had an intra-assay coefficient of variation $(\mathrm{CV})$ of $<10 \%$ with a coefficient of intraclass correlation of 0.98 . A subset of six samples was evaluated twice with a mean of 13 months in between, giving an inter-assay $\mathrm{CV}$ of $8.1 \%$ and further demonstrating the stability of the samples over time.

\section{Cardiorespiratory fitness}

Participants were familiarized with a treadmill previous to the maximal test. On the day of the test, they arrived at the exercise physiology laboratory in the afternoon wearing sports clothes, were connected to the gas analyzer and rested until their oxygen consumption $\left(\mathrm{VO}_{2}\right)$ values were below $4 \mathrm{~mL} \mathrm{O} / \mathrm{kg} \cdot \mathrm{min}$, in or- 
der to start all protocols from a similar condition close to basal metabolism. In a ramp ergospirometry treadmill protocol, participants warmed up for 3 minutes, walking at a speed of 3.5 miles/hr, with an inclination angle of $1 \%$. Following this, the speed of the treadmill was increased by $0.5 \mathrm{miles} / \mathrm{hr}$ every minute until exhaustion. Peak $\mathrm{VO}_{2}\left(\mathrm{VO}_{2 \text { peak }}\right)$ and carbon dioxide production $\left(\mathrm{VCO}_{2 \text { peak }}\right)$ were directly measured using an open circuit spirometer (Oxycon Delta; Jaeger, VIASYS Healthcare $\mathrm{GmbH}$, Höchberg, Germany), while the electrocardiogram and heart rate (HR) were continuously recorded. A maximum test was defined when (1) there was a plateau in $\mathrm{VO}_{2}$ despite an increase in work or a respiratory exchange ratio (RER) equal to or greater than 1.10 being observed; and (2) the participants reached the expected maximum $\mathrm{HR}$. The HR at minutes 1 and 5 of the recovery period was recorded.

\section{Body composition}

Dual-energy X-ray absorptiometry (DXA) was used to evaluate global and regional body composition $[33,34]$. Subjects arrived in the morning, in fasting conditions but well hydrated, as verified by a urinary specific gravity between 1,000 and 1,025, measured with a Brixco refractometer. DXA was performed in subjects wearing minimal clothes, using a Discovery Wi DXA system (Hologic, Middlesex County, MA, USA) and the Hologic APEX v4.5.3 software (Hologic). Visceral adipose tissue (VAT) was determined by subtracting the subcutaneous adipose tissue (SAT) from the total abdominal fat, as previously described [35]. Total abdominal fat was acquired from a rectangular region of interest, located between two horizontal lines placed at the L3-L4 space and the superior rib of the iliac crest as the upper and lower limits, respectively. DXA has shown excellent accuracy for measuring VAT in obese subjects of both sexes, compared to computed tomography [35] and magnetic resonance imaging as the gold standards [36]. Muscle mass of the right thigh was measured in an area located between two horizontal lines placed at the lowest point of the ischial tuberosity and at the knee joint as the upper and lower limits, respectively [37]. Other parameters reflecting global lean and fat mass were also obtained, as indicated in the Results section.

\section{Muscle composition}

The proportion of the area occupied by type II muscle fibers was estimated based on the quantification of carnosine in the right vastus lateralis muscle (VLM) using proton magnetic resonance spectroscopy [38] and a flexible coil [39], following the recommendations of the international consensus on spectrosco- py in skeletal muscle [40]. The VLM plays an important role in glycemic control and is associated with the development of metabolic diseases in humans [6,41]. Carnosine is a surrogate of muscle composition in humans, as validated in biopsied athletes [38] and obese/diabetic subjects [42].

Each individual arrived at the radiology service in the morning, and was placed in supine position on the spectroscopy table, with the lower limbs extended and supported on a 5-cm cushion in the popliteal region. A 3-T Magnetom Skyra magnet, with an SC Flex large 4-channels, 3T receive-only, 516×224 mm, coil interface, and the SyngoMR D13 program (Siemens Healthcare, Erlangen, Germany), were used to position a voxel of $15 \times 15 \times$ $35 \mathrm{~mm}^{3}$ in the right VLM (between the middle of the externally facing thigh and the middle third and distal junction of the same thigh), using a T1-weighted gradient-echo sequence in the three planes. The spectra were acquired using a point-resolved single voxel spectroscopy sequence with and without water saturation to evaluate the carnosine and water signals, respectively. The acquisition parameters were as follows: TE of $33 \mathrm{~ms}$, TR of 3,000 ms, averages of 192 for carnosine and 4 for water, 2,048 spectral data points, and a spectral bandwidth of 1,200 Hz. For signal processing, the jMRUI 5.1 software was used, with phase correction, Gaussian apodization, adjustment of the water reference to $4.7 \mathrm{ppm}$ and subtraction of water, lipids and other metabolites within the signal. Next, water and $\mathrm{C} 2-\mathrm{H}$ carnosine resonance integrals were estimated using the Advanced Method for Accurate, Robust and Efficient Spectral Fitting algorithm [43]. The absolute quantification of intramuscular carnosine concentration was performed following a published equation [44].

\section{Statistical analyses}

We used G*Power v3.1.9.7 (University of Düsseldorf, Düsseldorf, Germany) to calculate the sample size. Assuming a difference in musclin of $20 \%$ between groups, with a standard deviation (SD) of $25 \%$ within each group, an allocation ratio of 2 , an $\alpha$ of 0.05 , and a power of $85 \%$, we needed to enroll 27 subjects without and 55 with MS.

Data were analyzed using IBM SPSS software version 21.0 (IBM Corp., Armonk, NY, USA) and graphed in R packages (BoxPlotR and Corrplot). The Shapiro-Wilk test was performed to evaluate if data were normally distributed. Continuous variables were summarized using mean \pm SD for normally distributed data and median (interquartile range [IQR]) for variables with a skewed distribution. Percentages were used for categorical variables. The Mann-Whitney $U$ or Student's $t$ test was used to compare groups with and without MS, according to the data 
distribution. A logarithmic transformation was performed for musclin and HOMA-IR, and multiple linear regression models evaluating the relationship between the natural logarithm $(\ln )$ of musclin and $\ln (\mathrm{HOMA}-\mathrm{IR})$ or $\mathrm{VO}_{2 \text { peak, }}$, adjusted for age, sex, fat percentage, total lean mass, and physical activity, were carried out. Pearson correlation coefficients were used to evaluate the

Table 1. Demographics and Clinical Characteristics of the Subjects of the Study

\begin{tabular}{|c|c|c|c|}
\hline Variable & With metabolic syndrome $(n=61)$ & Without metabolic syndrome $(n=29)$ & $P$ value ${ }^{\mathrm{a}}$ \\
\hline \multicolumn{4}{|l|}{ Demographic } \\
\hline Female sex & $43(70.5)$ & $21(72.4)$ & 0.851 \\
\hline Age, yr & $50.7 \pm 6.1$ & $51.5 \pm 6.5$ & 0.691 \\
\hline \multicolumn{4}{|l|}{ Anthropometrics } \\
\hline Body mass index, $\mathrm{kg} / \mathrm{m}^{2}$ & $30.6 \pm 4.0$ & $27.4 \pm 3.6$ & $<0.010$ \\
\hline Body fat percentage, $\%$ & $38.8 \pm 7.0$ & $37.3 \pm 7.7$ & 0.252 \\
\hline Total lean mass/body mass $\times 100, \%$ & $57.3 \pm 6.8$ & $58.5 \pm 7.4$ & 0.444 \\
\hline Thigh lean mass/body mass $\times 100, \%$ & $6.2 \pm 0.8$ & $6.2 \pm 0.8$ & 0.935 \\
\hline Visceral adipose tissue, $\mathrm{g}$ & $812.1 \pm 252.1$ & $587.6 \pm 184.9$ & $<0.010$ \\
\hline Waist circumference, $\mathrm{cm}$ & $94.2(90.5-102.6)$ & $88.0(76.1-97.5)$ & $<0.010$ \\
\hline \multicolumn{4}{|l|}{ Cardiovascular } \\
\hline Systolic blood pressure, $\mathrm{mm} \mathrm{Hg}$ & $120.5 \pm 13.8$ & $119.4 \pm 15.7$ & 0.665 \\
\hline Diastolic blood pressure, $\mathrm{mm} \mathrm{Hg}$ & $76.7 \pm 10.0$ & $72.4 \pm 9.5$ & 0.047 \\
\hline Peak oxygen consumption, $\mathrm{mL} \mathrm{O}_{2} / \mathrm{kg} \cdot \min$ & $29.2 \pm 6.3$ & $31.0 \pm 5.8$ & 0.235 \\
\hline \multicolumn{4}{|l|}{ Habits } \\
\hline Smoking & $4(6.6)$ & $3(10.3)$ & 0.531 \\
\hline Alcohol consumption last month & $10(16.4)$ & $2(6.9)$ & 0.215 \\
\hline Physical activity, MET/hr/week & $8(2-17)$ & $4.3(0.4-32.6)$ & 0.340 \\
\hline \multicolumn{4}{|l|}{ Biochemical tests } \\
\hline Triglycerides, mg/dL & $182.7(136.8-219.9)$ & $106.9(86.0-132.5)$ & $<0.010$ \\
\hline High density cholesterol lipoprotein, mg/dL & $44.7 \pm 10.3$ & $50.9 \pm 11.6$ & 0.035 \\
\hline Glycemia, mg/dL & $98.9 \pm 8.7$ & $92.0 \pm 10.6$ & 0.029 \\
\hline Insulin, mIU/L & $14.5(11.4-19.3)$ & $7.0(5.1-8.2)$ & $<0.010$ \\
\hline Insulin resistance (HOMA-IR) & $3.5(2.9-4.6)$ & $1.7(1.1-2.0)$ & $<0.010$ \\
\hline \multicolumn{4}{|l|}{ Muscle composition } \\
\hline Carnosine, $\mathrm{mmol} / \mathrm{kg} \cdot \mathrm{ww}$ & $3.8 \pm 1.6$ & $3.3 \pm 1.5$ & 0.250 \\
\hline Area occupied by fibres type II, \% & $48.7 \pm 21.8$ & $41.7 \pm 19.7$ & 0.250 \\
\hline \multicolumn{4}{|l|}{ Medications } \\
\hline ACEI or ARA II & $20(32.8)$ & $3(10.3)$ & 0.023 \\
\hline Beta-blockers & $6(9.8)$ & 0 & 0.080 \\
\hline Calcium antagonists & $2(3.3)$ & 0 & 0.324 \\
\hline Diuretics & $11(18.0)$ & $1(3.4)$ & 0.057 \\
\hline Aspirin & $2(3.3)$ & 0 & 0.324 \\
\hline Statins & $10(16.4)$ & 0 & 0.021 \\
\hline Metformin & $2(3.3)$ & 0 & 0.324 \\
\hline
\end{tabular}

Values are expressed as number (\%), mean \pm standard deviation, or median (interquartile range).

MET, metabolic equivalent of task; HOMA-IR, homeostatic model assessment of insulin resistance; ww, wet weight; ACEI, angiotensin converting enzyme inhibitor; ARA II, angiotensin II receptor blocker.

${ }^{\mathrm{a} U n p a i r e d} t$ test. 
relationship among $\ln$ (musclin) and other variables. In all analyses, a two-sided $P<0.05$ was considered to indicate statistical significance.

\section{RESULTS}

\section{Comparisons between groups}

We included a total of 90 subjects, of whom 29 were controls and 61 had MS (Table 1). The groups were comparable across age, sex, indexes of lean mass, blood pressure, $\mathrm{VO}_{2 \text { peak, }}$ smoking, alcohol consumption, and weekly physical activity levels $(P>0.05)$. Although the participants with MS had higher carnosine content, and therefore a higher estimated area of type II fibers, the difference was not significant. The group with MS had higher BMI, VAT, waist circumference, triglyceridemia, glycemia, insulinemia, and HOMA-IR values $(P<0.05)$.

\section{Serum musclin}

The subjects with MS had higher median serum musclin values than the controls (206.7 pg/mL [IQR, 122.7 to 387.8$] \mathrm{vs.} 111.1 \mathrm{pg} / \mathrm{mL}$ [IQR, 63.2 to 218.5], $P<0.05$ ), as shown in Fig. 2.

This figure also shows that both groups partially overlapped at values around 50 to $300 \mathrm{pg} / \mathrm{mL}$, but there was a subgroup of individuals with MS who showed serum musclin concentrations over $300 \mathrm{pg} / \mathrm{mL}$. In order to better characterize this high-musclin subgroup, the whole sample was divided into tertiles according to the musclin concentrations and several variables were compared (Table 2). The subjects with higher musclin were those with higher HOMA-IR, visceral adiposity, and lean mass, but a lower body fat percentage. There were no differences regarding BMI, $\mathrm{VO}_{2 \text { peak, }}$ or level of physical activity.

\section{Correlation between serum musclin and IR}

A significant correlation was found between $\ln$ (musclin) and $\ln (\mathrm{HOMA}-\mathrm{IR})$ in a multiple linear regression model $(\beta=0.228$; $95 \%$ confidence interval $[\mathrm{CI}], 0.121$ to $0.334 ; P<0.01$ ) (Table 3 ) adjusted for age, sex, adiposity, lean mass, and level of physical activity.

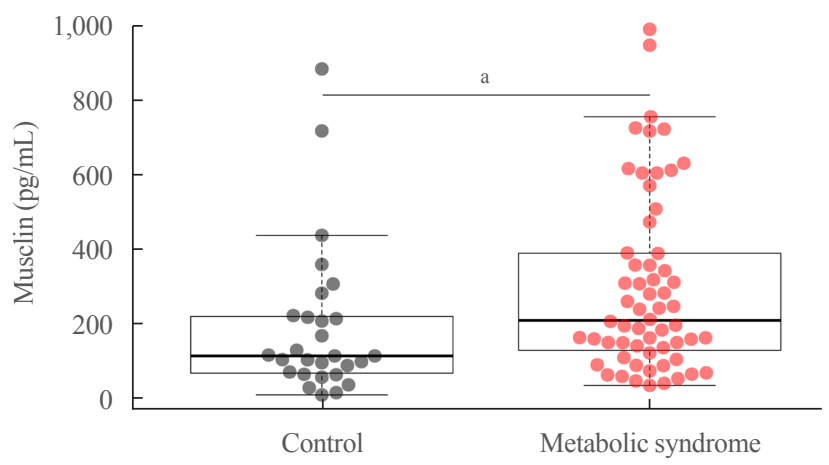

Fig. 2. Box plot showing the distribution of the serum musclin concentration in controls and subjects with metabolic syndrome. Each dot corresponds to a subject. The thicker horizontal lines correspond to the medians, whose values are presented in the text. ${ }^{a} P<0.05$ using the Mann-Whitney $U$ test.

Table 2. Tertiles of Musclin in Relation to Variables of Body Composition and Peak Oxygen Consumption

\begin{tabular}{|c|c|c|c|c|}
\hline & $\begin{array}{c}\text { Tertil } 1 \\
\text { (low musclin) } \\
63.9 \mathrm{pg} / \mathrm{mL}(45.3-91.4)\end{array}$ & $\begin{array}{c}\text { Tertil } 2 \\
\text { (intermediate musclin) } \\
\text { 173.1 pg/mL (147-212.3) }\end{array}$ & $\begin{array}{c}\text { Tertil } 3 \\
\text { (high musclin) } \\
489 \mathrm{pg} / \mathrm{mL}(334.4-716)\end{array}$ & $\begin{array}{c}P \text { value } \\
(\mathrm{T} 3 \text { vs. } \mathrm{T} 1+\mathrm{T} 2)\end{array}$ \\
\hline Age, yr & $49(43.3-53.8)$ & $52.5(47.8-57)$ & $53(48.3-58)$ & 0.08 \\
\hline HOMA-IR & $2.2(1.4-3.1)$ & $3.3(2.1-4.5)$ & $3.3(2.5-5.1)$ & 0.04 \\
\hline Body mass index, $\mathrm{kg} / \mathrm{m}^{2}$ & $29.6(27.3-31.4)$ & $29.8(25.4-32.6)$ & $28.8(26.5-32.8)$ & 0.76 \\
\hline Body fat percentage, $\%$ & $41.7(37.7-45.2)$ & $40.3(37.2-45)$ & $35.2(27.5-42.4)$ & $<0.01$ \\
\hline Visceral adipose tissue, $\mathrm{g}$ & $623.5(509.3-730.8)$ & $755(536.3-962.8)$ & $758.5(610.5-995.3)$ & 0.04 \\
\hline Waist circumference, $\mathrm{cm}$ & $88.5(82.9-97.9)$ & $92(84.3-102.9)$ & $95.4(92.2-104.1)$ & $<0.01$ \\
\hline Total lean mass corrected for stature, $\mathrm{kg} / \mathrm{m}^{2}$ & $22.6(21.3-24.6)$ & $22.4(21.5-24.1)$ & $26.2(23.3-28.5)$ & $<0.01$ \\
\hline Total lean mass corrected for body mass, $\mathrm{kg} / \mathrm{kg}$ & $0.5(0.5-0.6)$ & $0.6(0.5-0.6)$ & $0.6(0.5-0.7)$ & $<0.01$ \\
\hline $\mathrm{VO}_{2 \text { peak }}, \mathrm{mL} \mathrm{O}_{2} / \mathrm{kg} \cdot \min$ & $29.2(23.8-32.1)$ & $28.6(25.6-32.3)$ & $30.9(25.6-37.6)$ & 0.10 \\
\hline Physical activity, MET/hr/week & $3.67(0.33-11.0)$ & $7.3(3.4-16.7)$ & $7.33(1.33-36.0)$ & 0.66 \\
\hline
\end{tabular}




\section{Lack of correlation between serum musclin and cardiorespiratory capacity}

There was no correlation between $\ln$ (musclin) and $\mathrm{VO}_{2 \text { peak }}$ in a multiple linear regression model $(\beta=-0.287 ; 95 \% \mathrm{CI},-1.337$ to $0.762 ; P>0.05$ ) (Table 4 ) adjusted for age, sex, adiposity, lean mass, and level of physical activity. The association was not significant even when using the $\mathrm{VO}_{2 \text { peak }}$ corrected for total lean $\operatorname{mass}\left(\mathrm{VO}_{2 \text { peak }} / \mathrm{kg}\right)$.

\section{Pearson correlations}

We constructed two Pearson correlation matrices to further explore the relationship between musclin and metabolic (Fig. 3) or cardiorespiratory fitness (Fig. 4) variables. In agreement with the results shown in Tables $2,3, \ln$ (musclin) showed significant positive correlations with $\ln (\mathrm{HOMA}-\mathrm{IR}), \ln ($ insulin), glycemia, waist circumference, VAT, and thigh lean mass/total body mass $(P>0.05)$. Ln(musclin) did not correlate with the BMI or the area of type II fibers in the VLM. There were significant negative correlations between $\ln$ (musclin) and fat percentage and fat mass/stature squared index $(P>0.05)$ (Fig. 3).

Since the lack of correlation between musclin and BMI was noteworthy, we further explored the relationship between tertiles of BMI with cardiorespiratory capacity variables and serum musclin. The results confirmed that the tertile with the most

\begin{tabular}{|c|c|c|c|c|}
\hline $\begin{array}{l}\text { Dependent variable } \mathrm{Ln} \text { insulin resistance } \\
\text { (HOMA-IR) }(n=90)\end{array}$ & $\beta$ & $t$ & $P$ value & $95 \% \mathrm{CI}$ \\
\hline Constant & -5.866 & -4.604 & $<0.001$ & -8.401 to -3.331 \\
\hline Age, yr & -0.004 & -0.462 & 0.645 & -0.021 to 0.013 \\
\hline Female sex & 0.345 & 1.585 & 0.117 & -0.088 to 0.779 \\
\hline Fat percentage, $\%$ & 0.050 & 4.228 & $<0.001$ & 0.027 to 0.074 \\
\hline Total lean mass corrected for stature, $\mathrm{kg} / \mathrm{m}^{2}$ & 0.154 & 5.068 & $<0.001$ & 0.094 to 0.215 \\
\hline Physical activity, MET/hr/week & 0.000 & 0.275 & 0.784 & -0.002 to 0.002 \\
\hline Ln musclin, $\mathrm{pg} / \mathrm{mL}$ & 0.228 & 4.261 & $<0.001$ & 0.121 to 0.334 \\
\hline \multicolumn{5}{|c|}{$\begin{array}{l}\text { HOMA-IR, homeostatic model assessment of insulin resistance; CI, confidence interval; MET, metabolic equivalent of task. } \\
{ }^{a} \text { After adjusting for age, sex, fat percentage, total lean mass corrected for stature and physical activity, musclin was significantly associated with HOMA- } \\
\text { IR. These variables were selected according to biological plausibility and our results. The model showed an } R^{2}=0.397 \text {. The analysis of variance showed } \\
\text { that at least one coefficient was different from } 0(\mathrm{~F}=8.87 ; P<0.001) \text {. The tolerance }(>0.1) \text { and variance inflation factor }(<5) \text { showed that there was no } \\
\text { collinearity between the independent variables. The analysis of the residuals showed adherence to the assumptions of normality, homoscedasticity } \\
\text { (Breusch-Pagan test; } P>0.05 \text { ) and no autocorrelation (Durbin-Watson } \mathrm{d}=1.80 \text { ). }\end{array}$} \\
\hline
\end{tabular}

Table 4. Multiple Linear Regression Model Showing the Lack of Associations between Musclin and Peak Oxygen Consumption ${ }^{\mathrm{a}}$

\begin{tabular}{lcccc}
\hline $\begin{array}{l}\text { Dependent variable Peak oxygen consumption } \\
\left(\mathrm{mL} \mathrm{O}_{2} / \mathrm{kg} / \mathrm{min}\right)(n=90)\end{array}$ & $\beta$ & $t$ & \multicolumn{2}{c}{$P$ value } \\
\hline Constant & 88.106 & 7.010 & $<0.001$ & 63.099 to 113.114 \\
Age, $\mathrm{yr}$ & -0.045 & -0.532 & 0.596 & -0.211 to 0.122 \\
Female sex & -4.271 & -1.986 & 0.050 & -8.549 to 0.008 \\
Fat percentage, $\%$ & -0.717 & -6.131 & $<0.001$ & -0.949 to -0.484 \\
Total lean mass corrected for stature, $\mathrm{kg} / \mathrm{m}^{2}$ & -1.000 & -3.335 & 0.001 & -1.597 to -0.404 \\
Physical activity, $\mathrm{MET} / \mathrm{hr} /$ week & 0.001 & 0.151 & 0.881 & -0.017 to 0.020 \\
Ln musclin, $\mathrm{pg} / \mathrm{mL}$ & -0.287 & -0.545 & 0.587 & -1.337 to 0.762 \\
\hline
\end{tabular}

CI, confidence interval; MET, metabolic equivalent of task.

${ }^{a}$ After adjusting for age, sex, fat percentage, total lean mass corrected for stature and physical activity, musclin was not significantly associated with peak oxygen consumption. The variables incorporated in the model were selected according to biologic plausibility and our results. The model showed an $R^{2}=0.397$. The analysis of variance showed that at least one coefficient was different from $0(\mathrm{~F}=14.23 ; P<0.001)$. Tolerance $(>0.1)$ and the variance inflation factor $(<5)$ showed that there was no collinearity between the independent variables. The analysis of the residuals showed adherence to the assumptions of normality, homoscedasticity (Breusch-Pagan test; $P>0.05$ ) and no autocorrelation (Durbin-Watson $\mathrm{d}=2.37$ ). 


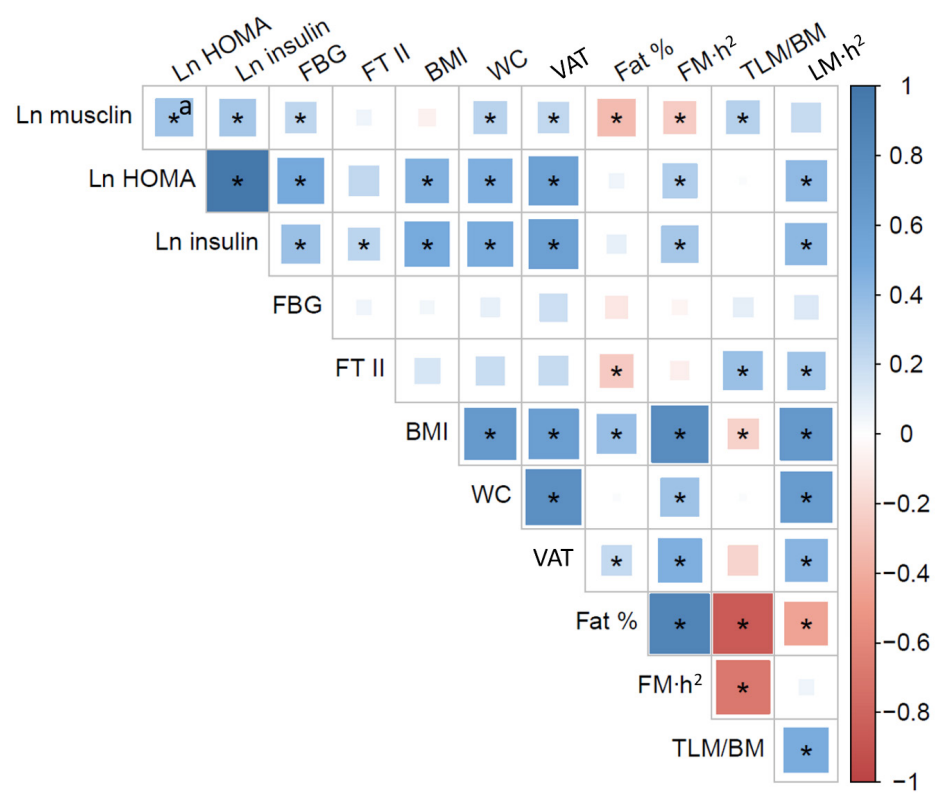

Fig. 3. Pearson correlation heatmap to assess the relationship between musclin and biochemical and body composition variables in humans. $\mathrm{Ln}$ (musclin) showed positive and significant correlations with biochemical variables such as ln(homeostatic model assessment of insulin resistance [HOMA-IR]) $(r=0.34), \ln ($ insulin $)(r=0.32)$ and fasting blood glucose $(\mathrm{FBG}, \mathrm{mg} / \mathrm{dL})(r=0.23)$. It also showed positive correlations with the parameters of abdominal obesity, waist circumference (WC, $\mathrm{cm})(r=0.25)$, and visceral adipose tissue (VAT, g) $(r=0.22)$. The correlation of musclin and parameters of global adiposity such as body fat percentage (fat \%) $(r=-0.33)$ and FM $(r=-0.25)$ was negative. Musclin showed a positive correlation with a measure of lean mass, thigh lean mass corrected for body mass $(\mathrm{TLM} / \mathrm{BM}, \mathrm{kg} / \mathrm{kg})(r=0.26), \mathrm{but}$ not to muscle fiber type composition, area of type II fibers (FT II, \%) $(r=0.05)$. There was no correlation between musclin and body mass index $\left(\mathrm{BMI}, \mathrm{kg} / \mathrm{m}^{2}\right)$. Other significant correlations are also highlighted. The strength of each association is indicated by the intensity of the color grades, according to the key shown on the right. $\mathrm{FM} \mathrm{h}^{2}$, fat mass corrected for stature $\left(\mathrm{kg} / \mathrm{m}^{2}\right)$; $\mathrm{LM} \mathrm{h}^{2}$, total lean mass corrected for stature $\left(\mathrm{kg} / \mathrm{m}^{2}\right) .{ }^{a} \mathrm{All} *$ indicate $P<0.05$.

obese subjects showed the lowest $\mathrm{VO}_{2 \text { peak }}$ and the lowest maximum time of work, but did not show musclin concentrations different from those of the other tertiles $(P>0.05)$ (not shown).

In accordance with the above results, there were no correlations between $\ln$ (musclin) and $\mathrm{VO}_{2 \text { peak, }} \mathrm{VO}_{\text {2peak }}$ corrected for body mass or lean mass, $\mathrm{VCO}_{2 \text { peak, }}$ maximum $\mathrm{HR}$, maximum velocity, maximum time of work, RER, or recovery HR $(P>0.05)$ (Fig. 4). There was also no correlation between $\ln$ (musclin) and weekly physical activity levels (not shown).

\section{DISCUSSION}

One view regarding the function of musclin proposes that it regulates glycemia and induces IR and metabolic alterations, while the other suggests that it improves physical performance without inducing metabolic disorders. The comparison between humans with and without MS has emerged as a testing ground to evaluate which of these two different views is more plausible from a physiological standpoint. We found that: (1) subjects with MS and IR had higher fasting serum musclin levels than subjects with neither MS nor IR; (2) serum musclin was positively correlated with IR and a body composition profile with high visceral adiposity and lean mass, but low body fat percentage; and (3) musclin did not correlate with BMI or any variable of cardiorespiratory fitness collected during an ergospirometry test.

\section{Musclin is higher in subjects with MS}

Muscle mass, muscle composition, and endocrine function have clear implications for the understanding of the pathophysiology of chronic metabolic diseases such as MS [13,14]. In terms of endocrine function, the physiological role of musclin in mammals has been the subject of controversy. Moreover, the study of the function of musclin in humans has lagged significantly when compared to studies in murine models.

Our results showed that subjects with MS had higher values of serum musclin. Furthermore, musclin showed positive correlations with IR and VAT, but negative correlations with indicators of total body fat. Total adiposity, as indicated by fat mass 


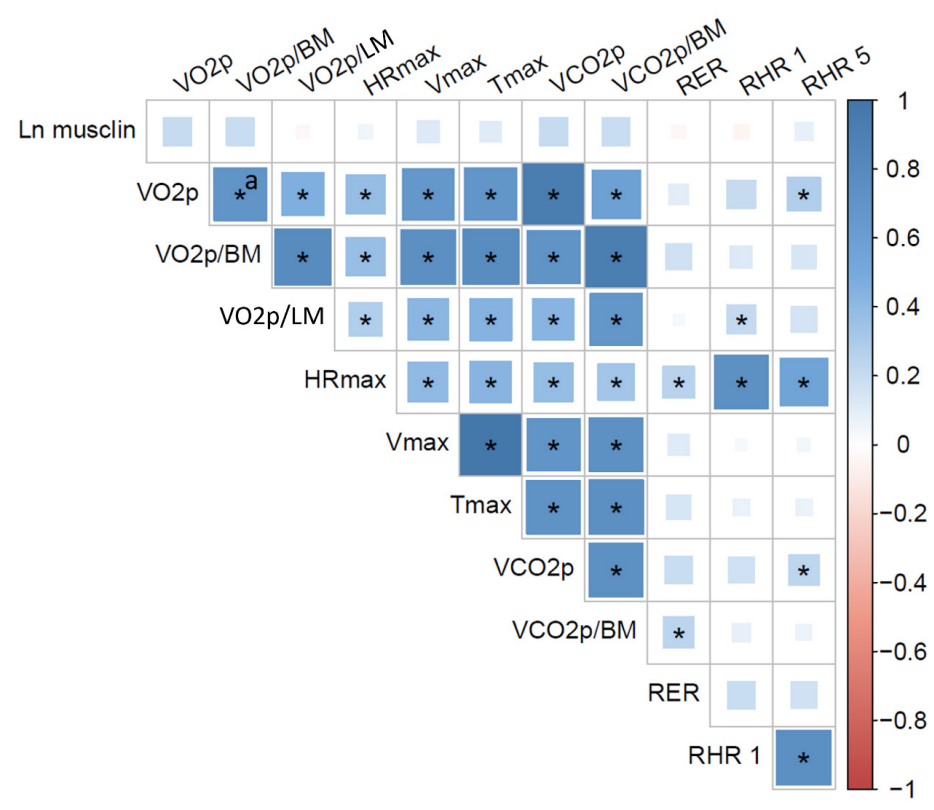

Fig. 4. Heatmap of Pearson correlations to assess the relationship between musclin and variables of physical capacity in humans. There was no correlation between $\ln$ (musclin) and any of the variables related to physical endurance and cardiorespiratory fitness obtained during the ergospirometry test $(P>0.05$ in all cases). The strength of each association is indicated by the intensity of the color grades, according to the key shown on the right. VO2p, $\mathrm{VO}_{2 \text { peak }}(\mathrm{mL} \mathrm{O} / \mathrm{min}) ; \mathrm{VO} 2 \mathrm{p} / \mathrm{BM}, \mathrm{VO}_{2 \text { peak }}$ corrected for body mass $(\mathrm{mL} \mathrm{O} / \mathrm{kg} \cdot \mathrm{min}) ; \mathrm{VO} 2 \mathrm{p} / \mathrm{LM}, \mathrm{VO}$ peak $\mathrm{cor}-$ rected for total lean mass $\left(\mathrm{mL} \mathrm{O}_{2} / \mathrm{kg} \cdot \mathrm{min}\right)$; HRmax, maximum heart rate (bpm); Vmax, maximum velocity (mile/hr); Tmax, maximum time of work (min); $\mathrm{VCO} 2 \mathrm{p}, \mathrm{VCO}_{2 \text { peak }}(\mathrm{mL} \mathrm{CO} / \mathrm{min}) ; \mathrm{VCO} 2 \mathrm{p} / \mathrm{BM}, \mathrm{VCO}_{2 \text { peak }}$ corrected for body mass $\left(\mathrm{mL} \mathrm{CO}_{2} / \mathrm{kg} \cdot \mathrm{min}\right)$; RER, respiratory ex-

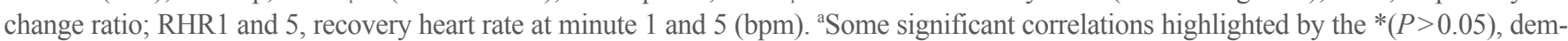
onstrate the reliability of the data.

and fat percentage measurements, mainly (approximately 80\%) reflects SAT. Although higher total adiposity is related to metabolic impairment, VAT is the adipose compartment that is more pathogenic and more strongly related to increased risks of MS and IR [45-49]. This observation was confirmed in our population, in which we observed a positive association between HOMA-IR and VAT $(r=0.58, P<0.05)$. Then, not only are VAT and SAT anatomically separated, but most importantly, they are developmentally and functionally distinct, which gives them different implications for the development of metabolic diseases [45-49]. For instance, the removal of VAT in murine models of obesity reversed IR and modified the profile of serum adipokines [50]. Moreover, mice that received a transplant of SAT into the VAT area improved insulin sensitivity [51], clearly demonstrating a different metabolic profile between VAT and SAT. The differences are further confirmed because VAT has more potential for inflammation (mainly by producing more tumor necrosis factor alpha, interleukin 6 , and monocyte chemoattractant protein-1), which reduces insulin sensitivity, and is also the main source of resistin, an adipokine proposed to favor IR and boost inflammation [46]. VAT has also long been shown to elicit a higher turnover of palmitate [52], which is an important marker and inducer of IR in cellular models [53]. Subjects with MS and IR have greater total free fatty acids and palmitate than obese subjects without IR [54,55]. Instead, SAT is the main source of adiponectin, an insulin-sensitizing, anti-inflammatory adipokine [46]. In subjects with high trunk fat (mainly VAT) and IR, those who also had large SAT values had higher adiponectin and lower inflammatory markers than those with less SAT [48]. This confirms that, in contrast to VAT, large amounts of SAT have favorable effects on adiponectin, inflammatory markers, IR, and MS risk.

Therefore, the subjects in our study with high SAT (i.e., high total fat mass and fat percentage), irrespective of their VAT, were expected to have higher adiponectin, as well as lower palmitate and inflammatory markers, a profile associated with less IR. In this manner, the inverse association between musclin and total fat can be explained if adiponectin inhibits the expression and secretion of musclin. Conversely, subjects with high musclin and high VAT, which in our study were those with MS, IR, and low SAT, were expected to have lower adiponectin levels but higher levels of resistin, palmitate and inflammatory markers. 
Thus, the fact that musclin was positively associated with VAT and IR can be explained if resistin, palmitate, and inflammatory markers (either in isolation or through the concerted action of all these molecules) favor musclin expression and secretion.

In agreement with this hypothesis, it was recently shown that palmitate activates the phospho-protein kinase RNA-like ER kinase (PERK)/activating transcription factor 4 (ATF4)/CCAAT/ enhancer-binding protein $(\mathrm{C} / \mathrm{EBP})$ signaling pathway in myotubes to increase the transcription of musclin [18], which may then appear in serum, as serum musclin concentrations seem to follow musclin intramuscular expression $[19,20]$. The specific relationship between musclin and adipokines, other free fatty acids, and inflammatory markers is an avenue that should be addressed in future basic and clinical studies.

\section{Musclin positively correlates with IR}

In agreement with the above discussion, we found that musclin correlated with IR after adjusting for age, sex, fat percentage, and lean mass. This observation agrees with a recent study in obese people [15]. However, in that paper, the authors did not assess body composition, lean mass, or muscle composition in detail. Moreover, the lean patients in that study had a HOMAIR over 3, which in our population corresponded to IR.

In the present study, we found that musclin positively correlated with HOMA-IR and visceral adiposity in participants who were clearly separated as with or without MS and IR (HOMAIR over or below the cutoff value of 2.25 , respectively), and this relationship remained consistent after adjusting for variables associated with body composition.

The lack of a correlation between musclin and muscle composition and the low magnitude of the correlation between musclin and muscle mass suggest that several circulating musclin isoforms might exist. Although skeletal muscle is the main source of musclin [11], this myokine is also produced by the bones [56] and the heart [23]. Even though it is not currently possible to distinguish the specific contribution of any of the isoforms, if they actually exist, we propose that our results mainly reflect muscle-derived musclin.

The results showed a positive correlation between musclin and insulinemia, in agreement with earlier experiments conducted in myotubes [11], which suggested that insulin is required for musclin to induce IR. Thus, it seems that in humans, the increased insulinemia associated with the presence of IR drives positive feedback that further favors the IR-inducing role of musclin.

\section{Musclin does not correlate with cardiorespiratory fitness}

In contrast to what could be extrapolated from some recent studies in mice, we did not find any association between musclin and variables related to cardiorespiratory capacity in untrained humans. Some data suggesting that musclin is a physical endurance enhancer were obtained in untrained musclin knockout (KO) mice [21]. Although those mice showed major bone and muscle parameters similar to the wild type, we speculate that musclin KO may have unnoticedly affected one or more cardiovascular, respiratory, musculoskeletal, or neurological molecular and epigenetic determinants of physical performance in mammals (including mice). Those determinants include, for instance, adrenergic signaling, calcium signaling, hemoglobin, myoglobin, lung reserve, alpha-actinin, mitochondrial function, cardiac contractile function, and capillary density [57]. Their single or combined alterations may explain possible differences in physical performance between wild-type and $\mathrm{KO}$ mice with healthy musculoskeletal development, and may become evident only under exercise or stress conditions, or through more indepth molecular studies.

In agreement with this, the musclin $\mathrm{KO}$ mice showed exacerbated muscle atrophy only when bearing cancer, indicating that several other regulators may be affected in this mouse model [22]. The existence of as yet unknown musclin isoforms, short forms, or several receptors for one or more isoforms, would also explain the dual function of this protein. In any case, the results obtained from musclin $\mathrm{KO}$ mice should be interpreted with caution until more knowledge about musclin-activated signaling pathways is gained and those animals are better characterized.

\section{Strengths and limitations}

We calculated a sample size with an allocation ratio of MS to control subjects of 2 instead of 1 , because control subjects have narrower ranges of musclin and HOMA-IR, which limits the power of the multiple and simple linear regression models. The subjects of both study groups were enrolled at the same time by the same researchers, and were evaluated by blinded researchers. Since the sample size was larger than required and the difference in musclin concentrations between groups was higher in magnitude than expected, we could estimate an actual study power of $96 \%$. There are also at least three reasons to argue against the possibility that the lack of correlations between musclin and cardiorespiratory capacity variables arose due to insufficient power: (1) the multiple regression model had a very high $P$ value, and even after adjustments the $95 \%$ confidence interval remained centered on zero; (2) none of the correlations showed a trend, 
which further suggests that type I errors were not present; and (3) several known correlations reported in Fig. 4 were significant, and the most obese subjects showed the worst cardiorespiratory capacity as expected, serving as quality controls and demonstrating that the sample was adequate. Thus, our good methodological design allowed us to test our hypotheses robustly.

About 3\% of participants with MS were on metformin. Since metformin reduces insulin, HOMA-IR, and IR [58], we speculate that metformin might have prevented the serum musclin in the MS group from being even higher and attenuated the correlations of musclin with insulin and HOMA-IR. Thus, the increased musclin values found in the subjects with MS are not explained by any known effect of metformin.

Since aging reduces the number, size and satellite cells of type II fibers [59], which are the main producers of musclin in mammals [16], we speculate that musclin may be reduced in older people. However, the effect of aging on musclin is not expected to affect our results because we: (1) enrolled only adults, not elderly subjects; (2) adjusted our regression analyses for age; (3) and matched groups with and without MS for age.

\section{Implications}

We provide evidence to show that the role of musclin in humans is in line with that shown in cell cultures and wild-type micethat is, musclin is likely involved in the regulation of glucose metabolism. Our results indicate that insulin, visceral and total adiposity, and muscle mass, but not BMI, fiber type, or physical fitness, are probably novel regulators of musclin in humans. The data supports a recent model proposed to integrate information on the role of skeletal muscle mass and its endocrine function in the pathophysiology of MS [13].

The evidence available up to now also allows us to propose musclin as a target to be regulated in patients with MS and IR. New public health policies should always consider and account for the role of the skeletal muscle as an important player in metabolic health issues.

\section{Conclusions}

In humans, musclin is positively associated with IR and with a body composition profile characterized by high visceral adiposity and lean mass, but low body fat percentage. This myokine is not related to BMI, cardiorespiratory fitness, or physical activity levels. The fact that most data obtained with cultured cells, wild-type murine models, and now in humans supports this conclusion suggests that the results obtained in $\mathrm{KO}$ mice should be interpreted carefully.

\section{CONFLICTS OF INTEREST}

No potential conflict of interest relevant to this article was reported.

\section{ACKNOWLEDGMENTS}

The study was funded by grants from Colciencias-Coldeportes (Convocatoria 626-2014, project 111562638757 from August 11, 2014), Colciencias (Beca de Doctorados Nacionales 727 2015), Convenio Interinstitucional IPS-Universitaria-Facultad de Medicina (minutes 01 from 6th July 2016, project 13041), CODI (minutes 54 from August 24, 2013, project 2565) and Fundación para la Promoción de la Investigación y la Tecnología (Banco de la República) (project 4.339 from March 12, 2019). The funders did not participate in data collection and analysis, article writing or submission.

We thank the postgraduate students Leonardo Castro, Esperanza Montoya, Laura Pérez, Daniel Restrepo, Oscar Guerrero, Sergio Cardona and Jorge Petro, from University of Antioquia (UdeA), and Iván Montoya from Pontifical Bolivarian University, for their support. We express special thanks to secretary Ana Arcia, her assistants, and professor Norman Balcázar from UdeA. We also thank the support received from patients, as well as students of the Physical Activity and Sports Medicine Postgraduate Program, Faculty of Medicine, Biochemical Laboratory, IPS-Universitaria, Institute of Physical Education and Epidemiology Group, all from UdeA, in Medellin, Colombia. Micheline Tamayo and physician Alexandra Montoya were very helpful with the spectra acquisition at the Pablo Tobón Uribe Hospital. We are indebted to Shyuan Ngo from The University of Queensland, Australia, for critical reading of the manuscript and help with English editing.

\section{AUTHOR CONTRIBUTIONS}

Conception or design: J.G.V., J.C.C. Acquisition, analysis, or interpretation of data: Y.L.S., M.Y.C., L.V., A.F.M., M.C.T.A., S.G., M.P., M.E.C., J.C.A., R.N.S., J.G.V., J.C.C. Drafting the work or revising: M.Y.C., J.G.V., J.C.C. Final approval of the manuscript: Y.L.S., M.Y.C., L.V., A.F.M., M.C.T.A., S.G., M.P., M.E.C., J.C.A., R.N.S., J.G.V., J.C.C.

\section{ORCID}

Yeliana L. Sánchez https://orcid.org/0000-0003-1643-4711 
Juan C. Calderón https://orcid.org/0000-0002-8695-6972

\section{REFERENCES}

1. DeFronzo RA. Lilly lecture 1987: the triumvirate: beta-cell, muscle, liver: a collusion responsible for NIDDM. Diabetes 1988;37:667-87.

2. Lauritzen HP, Galbo H, Toyoda T, Goodyear LJ. Kinetics of contraction-induced GLUT4 translocation in skeletal muscle fibers from living mice. Diabetes 2010;59:2134-44.

3. Lund S, Holman GD, Schmitz O, Pedersen O. Contraction stimulates translocation of glucose transporter GLUT4 in skeletal muscle through a mechanism distinct from that of insulin. Proc Natl Acad Sci U S A 1995;92:5817-21.

4. Atlantis E, Martin SA, Haren MT, Taylor AW, Wittert GA; Members of the Florey Adelaide Male Ageing Study. Inverse associations between muscle mass, strength, and the metabolic syndrome. Metabolism 2009;58:1013-22.

5. Jurca R, Lamonte MJ, Barlow CE, Kampert JB, Church TS, Blair SN. Association of muscular strength with incidence of metabolic syndrome in men. Med Sci Sports Exerc 2005; 37:1849-55.

6. Londono FJ, Calderon JC, Gallo J. Association between thigh muscle development and the metabolic syndrome in adults. Ann Nutr Metab 2012;61:41-6.

7. Park BS, Yoon JS. Relative skeletal muscle mass is associated with development of metabolic syndrome. Diabetes Metab J 2013;37:458-64.

8. Srikanthan P, Karlamangla AS. Relative muscle mass is inversely associated with insulin resistance and prediabetes: findings from the third National Health and Nutrition Examination Survey. J Clin Endocrinol Metab 2011;96:2898903.

9. Bostrom P, Wu J, Jedrychowski MP, Korde A, Ye L, Lo JC, et al. A PGC1- $\alpha$-dependent myokine that drives brown-fatlike development of white fat and thermogenesis. Nature 2012;481:463-8.

10. Liu Y, Huo X, Pang XF, Zong ZH, Meng X, Liu GL. Musclin inhibits insulin activation of Akt/protein kinase $\mathrm{B}$ in rat skeletal muscle. J Int Med Res 2008;36:496-504.

11. Nishizawa H, Matsuda M, Yamada Y, Kawai K, Suzuki E, Makishima M, et al. Musclin, a novel skeletal muscle-derived secretory factor. J Biol Chem 2004;279:19391-5.

12. Ahima RS, Park HK. Connecting myokines and metabolism. Endocrinol Metab (Seoul) 2015;30:235-45.

13. Narvaez-Sanchez R, Calderon JC, Vega G, Trillos MC,
Ospina S. Skeletal muscle as a protagonist in the pregnancy metabolic syndrome. Med Hypotheses 2019;126:26-37.

14. Kim G, Kim JH. Impact of skeletal muscle mass on metabolic health. Endocrinol Metab (Seoul) 2020;35:1-6.

15. Chen WJ, Liu Y, Sui YB, Yang HT, Chang JR, Tang CS, et al. Positive association between musclin and insulin resistance in obesity: evidence of a human study and an animal experiment. Nutr Metab (Lond) 2017;14:46.

16. Banzet S, Koulmann N, Sanchez H, Serrurier B, Peinnequin A, Bigard AX. Musclin gene expression is strongly related to fast-glycolytic phenotype. Biochem Biophys Res Commun 2007;353:713-8.

17. Tanner CJ, Barakat HA, Dohm GL, Pories WJ, MacDonald $\mathrm{KG}$, Cunningham PR, et al. Muscle fiber type is associated with obesity and weight loss. Am J Physiol Endocrinol Metab 2002;282:E1191-6.

18. Guo Q, Hu H, Liu X, Yang D, Yin Y, Zhang B, et al. C/ EBP $\beta$ mediates palmitate-induced musclin expression via the regulation of PERK/ATF4 pathways in myotubes. Am J Physiol Endocrinol Metab 2019;316:E1081-92.

19. Yu J, Zheng J, Liu XF, Feng ZL, Zhang XP, Cao LL, et al. Exercise improved lipid metabolism and insulin sensitivity in rats fed a high-fat diet by regulating glucose transporter 4 (GLUT4) and musclin expression. Braz J Med Biol Res 2016;49:e5129.

20. Deng Y, Tang Z. Research on intervention effects of musclin content in type 2 diabetic rats by aerobic exercise. Zhejiang Sport Sci 2012;34:113-5.

21. Subbotina E, Sierra A, Zhu Z, Gao Z, Koganti SR, Reyes S, et al. Musclin is an activity-stimulated myokine that enhances physical endurance. Proc Natl Acad Sci U S A 2015;112: 16042-7.

22. Re Cecconi AD, Forti M, Chiappa M, Zhu Z, Zingman LV, Cervo L, et al. Musclin, a myokine induced by aerobic exercise, retards muscle atrophy during cancer cachexia in mice. Cancers (Basel) 2019;11:1541.

23. Jeremic N, Weber GJ, Theilen NT, Tyagi SC. Cardioprotective effects of high-intensity interval training are mediated through microRNA regulation of mitochondrial and oxidative stress pathways. J Cell Physiol 2020;235:5229-40.

24. Alberti KG, Eckel RH, Grundy SM, Zimmet PZ, Cleeman JI, Donato KA, et al. Harmonizing the metabolic syndrome: a joint interim statement of the International Diabetes Federation Task Force on Epidemiology and Prevention; National Heart, Lung, and Blood Institute; American Heart Association; World Heart Federation; International Atheroscle- 
rosis Society; and International Association for the Study of Obesity. Circulation 2009;120:1640-5.

25. Gallo Villegas JA, Ochoa Munera JE, Balparda Arias JK, Aristizabal Ocampo D. Cut points of waist circumference to identify subjects with insulin resistance in a colombian population. Acta Med Colomb 2013;38: 118-6.

26. Gallo-Villegas J, Aristizabal JC, Estrada M, Valbuena LH, Narvaez-Sanchez R, Osorio J, et al. Efficacy of high-intensity, low-volume interval training compared to continuous aerobic training on insulin resistance, skeletal muscle structure and function in adults with metabolic syndrome: study protocol for a randomized controlled clinical trial (Intraining-MET). Trials 2018;19:144.

27. Bull FC, Maslin TS, Armstrong T. Global physical activity questionnaire (GPAQ): nine country reliability and validity study. J Phys Act Health 2009;6:790-804.

28. Mancia G, De Backer G, Dominiczak A, Cifkova R, Fagard R, Germano G, et al. 2007 Guidelines for the management of arterial hypertension: the task force for the management of arterial hypertension of the European Society of Hypertension (ESH) and of the European Society of Cardiology (ESC). J Hypertens 2007;25:1105-87.

29. Wallace TM, Levy JC, Matthews DR. Use and abuse of HOMA modeling. Diabetes Care 2004;27:1487-95.

30. Jelleyman C, Yates T, O'Donovan G, Gray LJ, King JA, Khunti K, et al. The effects of high-intensity interval training on glucose regulation and insulin resistance: a metaanalysis. Obes Rev 2015;16:942-61.

31. Mattioni Maturana F, Martus P, Zipfel S, Nieb AM. Effectiveness of HIIE versus MICT in improving cardiometabolic risk factors in health and disease: a meta-analysis. Med Sci Sports Exerc 2021;53:559-73.

32. Su L, Fu J, Sun S, Zhao G, Cheng W, Dou C, et al. Effects of HIIT and MICT on cardiovascular risk factors in adults with overweight and/or obesity: a meta-analysis. PLoS One 2019;14:e210644.

33. Moreira OC, Alonso-Aubin DA, Patrocinio de Oliveira CE, Candia-Lujan R. Methods of assessment of body composition: an updated review of description, application, advantages and disadvantages. Arch Med Dep 2015;32:387-94.

34. Sutter T, Duboeuf F, Chapurlat R, Cortet B, Lespessailles E, Roux JP. DXA body composition corrective factors between Hologic Discovery models to conduct multicenter studies. Bone 2021;142:115683.

35. Micklesfield LK, Goedecke JH, Punyanitya M, Wilson KE, Kelly TL. Dual-energy X-ray performs as well as clinical computed tomography for the measurement of visceral fat. Obesity (Silver Spring) 2012;20:1109-14.

36. Neeland IJ, Grundy SM, Li X, Adams-Huet B, Vega GL. Comparison of visceral fat mass measurement by dual-Xray absorptiometry and magnetic resonance imaging in a multiethnic cohort: the Dallas Heart Study. Nutr Diabetes 2016;6:e221.

37. Visser M, Fuerst T, Lang T, Salamone L, Harris TB. Validity of fan-beam dual-energy X-ray absorptiometry for measuring fat-free mass and leg muscle mass: Health, Aging, and Body Composition Study. Dual-Energy X-ray Absorptiometry and Body Composition Working Group. J Appl Physiol (1985) 1999;87:1513-20.

38. Baguet A, Everaert I, Hespel P, Petrovic M, Achten E, Derave W. A new method for non-invasive estimation of human muscle fiber type composition. PLoS One 2011;6: e21956.

39. Estrada M, Vega G, Reyngoudt H, Ricaurte G, Cardona $\mathrm{OM}$, Gallo J, et al. Intramuscular absolute carnosine quantification in young athletes by $1 \mathrm{H}-\mathrm{MRS}$ using a flexible coil. Skelet Radiol 2016;45:1021-9.

40. Krssak M, Lindeboom L, Schrauwen-Hinderling V, Szczepaniak LS, Derave W, Lundbom J, et al. Proton magnetic resonance spectroscopy in skeletal muscle: experts' consensus recommendations. NMR Biomed 2021;34:e4266.

41. Olsen DB, Sacchetti M, Dela F, Ploug T, Saltin B. Glucose clearance is higher in arm than leg muscle in type 2 diabetes. J Physiol 2005;565(Pt 2):555-62.

42. Stegen S, Everaert I, Deldicque L, Vallova S, de Courten B, Ukropcova B, et al. Muscle histidine-containing dipeptides are elevated by glucose intolerance in both rodents and men. PLoS One 2015;10:e0121062.

43. Vanhamme L, van den Boogaart A, Van Huffel S. Improved method for accurate and efficient quantification of MRS data with use of prior knowledge. J Magn Reson 1997;129: 35-43.

44. Just Kukurova I, Valkovic L, Ukropec J, de Courten B, Chmelik M, Ukropcova B, et al. Improved spectral resolution and high reliability of in vivo (1) $\mathrm{H}$ MRS at $7 \mathrm{~T}$ allow the characterization of the effect of acute exercise on carnosine in skeletal muscle. NMR Biomed 2016;29:24-32.

45. Luong Q, Huang J, Lee KY. Deciphering white adipose tissue heterogeneity. Biology (Basel) 2019;8:23.

46. Chait A, den Hartigh LJ. Adipose tissue distribution, inflammation and its metabolic consequences, including diabetes and cardiovascular disease. Front Cardiovasc Med 2020;7:22. 
47. Fox CS, Massaro JM, Hoffmann U, Pou KM, MaurovichHorvat P, Liu CY, et al. Abdominal visceral and subcutaneous adipose tissue compartments: association with metabolic risk factors in the Framingham Heart Study. Circulation 2007;116:39-48.

48. Wu H, Qi Q, Yu Z, Sun Q, Wang J, Franco OH, et al. Independent and opposite associations of trunk and leg fat depots with adipokines, inflammatory markers, and metabolic syndrome in middle-aged and older Chinese men and women. $\mathrm{J}$ Clin Endocrinol Metab 2010;95:4389-98.

49. Zhang M, Hu T, Zhang S, Zhou L. Associations of different adipose tissue depots with insulin resistance: a systematic review and meta-analysis of observational studies. Sci Rep 2015;5:18495.

50. Gabriely I, Ma XH, Yang XM, Atzmon G, Rajala MW, Berg $\mathrm{AH}$, et al. Removal of visceral fat prevents insulin resistance and glucose intolerance of aging: an adipokine-mediated process? Diabetes 2002;51:2951-8.

51. Tran TT, Yamamoto Y, Gesta S, Kahn CR. Beneficial effects of subcutaneous fat transplantation on metabolism. Cell Metab 2008;7:410-20.

52. Jensen MD, Haymond MW, Rizza RA, Cryer PE, Miles JM. Influence of body fat distribution on free fatty acid metabolism in obesity. J Clin Invest 1989;83:1168-73.

53. Chavez JA, Summers SA. Characterizing the effects of satu- rated fatty acids on insulin signaling and ceramide and diacylglycerol accumulation in 3T3-L1 adipocytes and C2C12 myotubes. Arch Biochem Biophys 2003;419:101-9.

54. Bermudez-Cardona J, Velasquez-Rodriguez C. Profile of free fatty acids and fractions of phospholipids, cholesterol esters and triglycerides in serum of obese youth with and without metabolic syndrome. Nutrients 2016;8:54.

55. Boden G. Obesity and free fatty acids. Endocrinol Metab Clin North Am 2008;37:635-46.

56. Thomas G, Moffatt P, Salois P, Gaumond MH, Gingras R, Godin E, et al. Osteocrin, a novel bone-specific secreted protein that modulates the osteoblast phenotype. J Biol Chem 2003;278:50563-71.

57. Bouchard C, Hoffman E. Genetic and molecular aspects of sport performance. 18th ed. Chichester: Blackwell Publishing; 2011. Chapter 16, Genetic determinants of exercise performance: evidence from transgenic and null mouse models; p. 185-94.

58. He L. Metformin and systemic metabolism. Trends Pharmacol Sci 2020;41:868-81.

59. Verdijk LB, Koopman R, Schaart G, Meijer K, Savelberg $\mathrm{HH}$, van Loon LJ. Satellite cell content is specifically reduced in type II skeletal muscle fibers in the elderly. Am J Physiol Endocrinol Metab 2007;292:E151-7. 